\title{
FEEDBACK STABILIZATION OF NAVIER-STOKES EQUATIONS
}

\author{
VIOREL BARBU ${ }^{1}$
}

\begin{abstract}
One proves that the steady-state solutions to Navier-Stokes equations with internal controllers are locally exponentially stabilizable by linear feedback controllers provided by a $L Q$ control problem associated with the linearized equation.
\end{abstract}

Mathematics Subject Classification. 76D05, 76D55, 35B40, 35Q30.

Received July 14, 2002. Revised November 25, 2002.

\section{INTRODUCTION}

Consider the controlled Navier-Stokes system with the non-slip Dirichlet boundary conditions

$$
\begin{aligned}
& y_{t}(x, t)-\nu \Delta y(x, t)+(y \cdot \nabla) y(x, t)=m(x) u(x, t)+f_{0}(x)+\nabla p(x, t), \quad(x, t) \in Q \\
& (\nabla \cdot y)(x, t)=0, \quad \forall(x, t) \in Q=\Omega \times(0, \infty) \\
& y=0, \quad \text { on } \Sigma=\partial \Omega \times(0, \infty) \\
& y(x, 0)=y_{0}(x), \quad x \in \Omega .
\end{aligned}
$$

These equations govern the motion of viscous incompressible flows in a domain $\Omega$ of $R^{d}, d=2$ or $d=3$ where $y=\left(y_{1}, \ldots, y_{d}\right)$ is the state, the velocity field $u=\left(u_{1}, u_{2}, \ldots, u_{d}\right)$ is the control input and $p$ is the unknown pressure. Here $m$ is the characteristic function of an open subset $\omega$ of $\Omega$, and $f_{0}, y_{0} \in\left(L^{2}(\Omega)\right)^{d}, \nabla \cdot y_{0}=0$ are given vector fields.

Denote by $n$ the normal to $\partial \Omega$ and set

$$
H=\left\{y \in\left(L^{2}(\Omega)\right)^{d}, \nabla \cdot y=0, y \cdot n=0 \text { on } \partial \Omega\right\}, V=\left\{y \in\left(H_{0}^{1}(\Omega)\right)^{d}, \nabla \cdot y=0\right\} .
$$

Denote by $P:\left(L^{2}(\Omega)\right)^{d} \longrightarrow H$ the Leray projector and set

$$
b(y, z, w)=\sum_{i, j=1}^{d} \int_{\Omega} y_{i} D_{i} z_{j} w_{j} \mathrm{~d} x
$$

Define the operator $B: V \longrightarrow V^{\prime}$ by

$$
(B y, w)=b(y, y, w), \forall y, w \in V .
$$

Keywords and phrases. Navier-Stokes system, Riccati equation, linearized system, steady-state solution, weak solution.

1 Department of Mathematics, "Al.I. Cuza" University, 6600 Iaşi, Romania; e-mail: barbu@uaic.ro 
Then we may rewrite equation (1.1) as

$$
\begin{aligned}
& \frac{\mathrm{d} y}{\mathrm{~d} t}(t)+\nu A y(t)+B y(t)=P(m u)+P f_{0}, \quad t \in[0, \infty) \\
& y(0)=y_{0}
\end{aligned}
$$

where $A \in L\left(V, V^{\prime}\right)$ (the Stokes operator) is defined by

$$
(A y, w)=\sum_{i=1}^{d} \int_{\Omega} \nabla y_{i} \cdot \nabla w_{i} \mathrm{~d} x, \forall y, w \in V
$$

Let $\left(y_{e}, p_{e}\right)$ be a steady-state solution to (1.1), i.e.,

$$
\begin{array}{ll}
-\nu \Delta y_{e}+\left(y_{e} \cdot \nabla\right) y_{e}=\nabla p_{e}+f_{0}(x) & \text { in } \Omega \\
\nabla \cdot y_{e}=0 & \text { in } \Omega \\
y_{e}=0 & \text { on } \partial \Omega
\end{array}
$$

Throughout this paper we shall assume that the boundary $\partial \Omega$ is a finite union of $d-1$ dimensional $C^{\infty}$-connected manifolds diffeomorphic with $S_{d}^{r}=\left\{x \in R^{d},|x|=r\right\}$.

It is well known that for $d=2,3$ always there is a steady-state solution and for small viscosity constant $\nu$ this solution might be instable. However, by some recent results in [14,15] (see also [3]) if $\left(y_{e}, p_{e}\right)$ and $y_{0}$ are sufficiently smooth, for instance if

$$
\left.\left(y_{e}, p_{e}\right) \in\left(\left(H^{3}(\Omega)\right)^{d}\right) \cap V\right) \times H^{1}(\Omega), y_{0} \in\left(H^{2}(\Omega)\right)^{d} \cap V
$$

and $\left\|y_{0}-y_{e}\right\|_{\left(H^{2}(\Omega)\right)^{d}} \leq \eta$ is sufficiently small then for each $T>0$ there are

$$
u \in H^{1}\left(0, T ;\left(L^{2}(\Omega)\right)^{d}\right), y \in L^{\infty}\left(0, T ;\left(H^{2}(\Omega)\right)^{d} \cap V\right) \cap H^{1}(0, T ; H)
$$

and $p \in L^{2}\left(0, T ; H^{1}(\Omega)\right)$ satisfying $(1.1)$ and such that $y(x, T) \equiv y_{e}(x)$. (In 2-D similar exact controllability results were previously obtained in $[11,13]$.) In particular, this implies that there is a controller $u$ which stabilizes the steady-state solution $y_{e}$.

Here we shall use a different approach to stabilization inspired by Liapunov stability theory for finite dimensional systems. One must recall that a key element in stabilization of nonlinear ordinary differential systems is the linear feedback controller stabilizing the linearized system, usually, provided by an algebraic Riccati equation associated with an infinite horizon $L Q$ problem. However in the case of infinite dimensional systems with unbounded nonlinearities, as is the case here, the situation is more complicated and our goal is to show (see Th. 1 below) that this approach still works with an appropriate $L Q$ problem. This will allow to solve the local exponential stabilization problem for the Navier-Stokes using the solution of an appropriate algebraic Riccati equation associated with the linearized Stokes equation. As seen below the technique is applicable to a larger class of nonlinear evolution equations and in particular to parabolic semilinear equations.

For recent results on stabilization of fluid flows we refer to the works $[1,6,7,9,12]$ and the references given there.

Here and throughout in the sequel $H^{k}(\Omega)$ and $H^{1}(0, T ; X)(X$ is a Hilbert space) are usual Sobolev spaces on $\Omega$ and $(0, T)$, respectively. We shall denote by the same symbol $|\cdot|$ the norm of $H$ and of $\left(L^{2}(\Omega)\right)^{d}$. We shall denote by $\|\cdot\|$ the norm of $V$ and by $(\cdot, \cdot)$ the pairing between $V, V^{\prime}$ (the dual space of $V$ ) and, respectively, the scalar product of $H$. Finally $|\cdot|_{s}$ is the norm of the Sobolev space $\left(H^{s}(\Omega)\right)^{d}$. 


\section{Stabilization of the Linearized Equation}

Substituting $y$ by $y+y_{e}$ and $p$ by $p+p_{e}$ into equation (1.1) we are lead to the null stabilization of the equation

$$
\begin{array}{ll}
y_{t}-\nu \Delta y+(y \cdot \nabla) y+\left(y_{e} \cdot \nabla\right) y+(y \cdot \nabla) y_{e}=m u+\nabla p & \text { in } Q \\
\nabla \cdot y=0 & \text { in } Q \\
y=0 & \text { on } \Sigma \\
y(x, 0)=y_{0}(x)-y_{e}(x)=y^{0}(x), & x \in \Omega .
\end{array}
$$

Equivalently,

$$
\begin{aligned}
& \frac{\mathrm{d} y}{\mathrm{~d} t}(t)+\nu A y(t)+A_{0} y(t)+B y(t)=P(m u), t \geq 0 \\
& y(0)=y^{0}
\end{aligned}
$$

where $B, A$ are given by $(1.3,1.4)$ and $A_{0} \in L(V, H)$ is defined by

$$
\left(A_{0} y, w\right)=b\left(y_{e}, y, w\right)+b\left(y, y_{e}, w\right), \forall w \in H
$$

Consider the linearized system

$$
\begin{aligned}
& \frac{\mathrm{d} y}{\mathrm{~d} t}(t)+\nu A y(t)+A_{0} y(t)=P(m u)(t), t \geq 0 \\
& y(0)=y^{0}
\end{aligned}
$$

and the corresponding LQ(linear quadratic) optimal control problem

$$
\varphi\left(y^{0}\right)=\operatorname{Min}\left\{\frac{1}{2} \int_{0}^{\infty}\left(\left|A^{\frac{3}{4}} y(t)\right|^{2}+|u(t)|^{2}\right) \mathrm{d} t ; \text { subject to }(2.4)\right\} .
$$

We shall denote by $D(\varphi)$ the set of all $y^{0} \in H$ such that $\varphi\left(y^{0}\right)<\infty$ and note that for each $y^{0} \in D\left(A^{\frac{1}{4}}\right)$ the linear Stokes equation (2.4) is exactly null controllable on each interval $[0, T]$. More precisely, there are $u \in$ $L^{2}\left(0, T ;\left(L^{2}(\Omega)\right)^{d}\right), y \in L^{2}\left(0, T ; D\left(A^{\frac{3}{4}}\right)\right)$ satisfying $(2.4)$ and such that $y(T) \equiv 0$. Here is the argument. Let $y^{0} \in$ $D\left(A^{\frac{1}{4}}\right)$ and $0<T_{0}<T$. Then clearly equation (2.4) with $u=0$ has a unique strong solution

$$
y \in L^{2}\left(0, T_{0} ; D\left(A^{\frac{3}{4}}\right)\right), t^{\frac{1}{2}} A y \in L^{2}\left(0, T_{0} ; H\right) .
$$

Indeed by (2.4) we get the following a priori estimates

$$
\begin{aligned}
\frac{\mathrm{d}}{\mathrm{d} t}\left|A^{\frac{1}{4}} y\right|^{2}+2 \nu\left|A^{\frac{3}{4}} y\right|^{2} & =-2 b\left(y_{e}, y, A^{\frac{1}{2}} y\right)-2 b\left(y, y_{e}, A^{\frac{1}{2}} y\right)+2\left(m u, A^{\frac{1}{2}} y\right) \\
& \leq C\left|A^{\frac{1}{2}} y\right|\left(\left|y_{e}\right|_{2}\|y\|+|u|\right), \text { a.e. } t>0 .
\end{aligned}
$$

This yields

$$
\left|A^{\frac{1}{4}} y(t)\right|^{2}+\int_{0}^{t}\left|A^{\frac{3}{4}} y\right|^{2} \mathrm{~d} s \leq C\left(\int_{0}^{t}|u|^{2} \mathrm{~d} s+\left|A^{\frac{1}{4}} y^{0}\right|^{2}\right) .
$$

Next if multiply (2.4) by $t A y(t)$ and use the latter estimate we obtain after a similar calculation that

$$
\int_{0}^{t} s|A y(s)|^{2} \mathrm{~d} s \leq C\left(\int_{0}^{t}|u|^{2} \mathrm{~d} s+\left|A^{\frac{1}{4}} y^{0}\right|^{2}\right)
$$


which implies the desired result by standard argument. Now, if multiply the equation by $t A^{2} y$ we find that $t|A y(t)|^{2} \in C\left(\left(0, T_{0}\right]\right.$ and therefore $y\left(T_{0}\right) \in\left(H^{2}(\Omega)\right)^{d}$. Finally, by the controllability results established in $[14,15]$ (see also Lem. 3.1 in [3]) we know that there is a solution $(y, u)$ to $(2.4)$ on $\left(T_{0}, T\right)$ such that

$$
u \in H^{1}\left(T_{0}, T ;\left(L^{2}(\Omega)\right)^{d}\right), y \in L^{2}\left(T_{0}, T ; D(A)\right), y(T)=0 .
$$

In particular, this implies that $\varphi\left(y^{0}\right)<\infty, \forall y^{0} \in D\left(A^{\frac{1}{4}}\right)$ and more precisely, we have

$$
\varphi\left(y^{0}\right) \leq C\left|A^{\frac{1}{4}} y^{0}\right|^{2}, \forall y^{0} \in D\left(A^{\frac{1}{4}}\right) .
$$

Moreover, we have

$$
\varphi\left(y^{0}\right) \geq C\left|A^{\frac{1}{4}} y^{0}\right|^{2}
$$

Indeed, it is easily seen that for each $y^{0} \in D(\varphi)$ problem (2.5) has a unique solution $\left(y^{*}, u^{*}\right) \in L^{2}\left(R^{+} ; D\left(A^{\frac{3}{4}}\right)\right)$ $\times L^{2}\left(R^{+} ;\left(L^{2}(\Omega)\right)^{d}\right)$. Moreover, as easily seen by $(2.4), y^{*} \in C_{w}\left(R^{+}, D\left(A^{\frac{1}{4}}\right)\right)$. (Here $C_{w}$ denotes the space of weakly continuous functions.) If multiply equation (2.4) where $y=y^{*}, u=u^{*}$ by $A^{\frac{1}{2}} y^{*}$ and integrate on $(0, \infty)$ we obtain

$$
\begin{aligned}
\frac{1}{2}\left|A^{\frac{1}{4}} y^{0}\right|^{2} & \leq \int_{0}^{\infty}\left(\nu\left(A y^{*}, A^{\frac{1}{2}} y^{*}\right)+\left(A_{0} y^{*}, A^{\frac{1}{2}} y^{*}\right)+\left|u^{*}\right|\left|A^{\frac{1}{2}} y^{*}\right|\right) \mathrm{d} t \\
& \leq C \int_{0}^{\infty}\left(\left|A^{\frac{3}{4}} y^{*}\right|^{2}+\left|u^{*}\right|^{2}\right) \mathrm{d} t=C \varphi\left(y^{0}\right)
\end{aligned}
$$

because (see e.g. $[10,17])$ we have

$$
\left|\left(A_{0} y, A^{\frac{1}{2}} y\right)\right| \leq\left|b\left(y, y_{e}, A^{\frac{1}{2}} y\right)\right|+\left|b\left(y_{e}, y, A^{\frac{1}{2}} y\right)\right| \leq C\|y\|\left|A^{\frac{1}{2}} y\right|\left|y_{e}\right|_{2} \leq C\|y\|^{2}\left|y_{e}\right|_{2} .
$$

By (2.7) we may infer therefore that $D(\varphi)=D\left(A^{\frac{1}{4}}\right)=W$. The space $W$ is endowed with the graph norm $|y|_{W}=$ $\left|A^{\frac{1}{4}} y\right|$. Here and everywhere in the sequel $A^{s}, s \in(0,1)$, is the fractional power of the Stokes operator $A$ and $A^{s}=A^{[s]} A^{s-[s]}$ for $s \geq 1$. We refer to [10] for definition and basic properties. Here we recall only that

$$
V=D\left(A^{\frac{1}{2}}\right), D\left(A^{s}\right) \subset\left(H^{2 s}\right)^{d} \cap H, \forall s \geq 0
$$

and $\left(H^{m}(\Omega)\right)^{d} \cap V \subset D\left(A^{\frac{m}{2}}\right)$ for all positive integers $m$.

Since the function $\varphi$ is quadratic we may infer that there is a linear self-adjoint operator $R: H \rightarrow H$ with the domain $D(R)$ such that

$$
\frac{1}{2}\left(R y^{0}, y^{0}\right)=\varphi\left(y^{0}\right), \forall y^{0} \in D(R) \subset W .
$$

Moreover, $R \in L\left(W, W^{\prime}\right)$ and the latter equality extends to all of $W$.

Proposition 1. Let $d=2,3$. Then the optimal control $u^{*}$ is expressed as

$$
u^{*}(t)=-m R y^{*}(t), \forall t>0 .
$$

Moreover, $V \subset D(R)$, i.e.,

and there are $\omega_{i}>0, i=1,2$ such that

$$
|R y| \leq C\|y\|, \forall y \in V
$$

$$
\omega_{1}\|y\|_{W}^{2} \leq(R y, y) \leq \omega_{2}\|y\|_{W}^{2}, \quad \forall y \in W .
$$


The operator $R$ is a solution to the algebraic Riccati equation

$$
\left(\nu A y+A_{0} y, R y\right)+\frac{1}{2}|m R y|^{2}=\frac{1}{2}\left|A^{\frac{3}{4}} y\right|^{2}, \forall y \in D(A) .
$$

Proof. Estimate (2.10) follows by (2.6) and (2.7). Since the quadratic cost functional (2.5) is unbounded on $H$ the conclusions of Proposition 1 are not directly implied by the general theory of infinite dimensional LQ control problems (see e.g. $[8,16]$ ) and so it requires a direct treatment briefly presented below.

By the dynamic programming principle (see e.g. [2]) it follows that for each $T>0,\left(y^{*}, u^{*}\right)$ is the solution to optimal control problem

$$
\operatorname{Min}\left\{\frac{1}{2} \int_{0}^{T}\left(\left|A^{\frac{3}{4}} y(t)\right|^{2}+|u(t)|^{2}\right) \mathrm{d} t+\varphi(y(T)) ;(y, u) \text { subject to (2.4) }\right\} .
$$

Thus by the maximum principle we have that (see [2])

$$
u^{*}(t)=m q^{T}(t), \forall t \in[0, T)
$$

where $q^{T} \in L^{2}(0, T ; H) \cap C_{w}\left([0, T] ; V^{\prime}\right)$ is the solution to the adjoint equation

$$
\begin{aligned}
& \frac{\mathrm{d}}{\mathrm{d} t} q^{T}-\left(\nu A+A_{0}\right)^{*} q^{T}=A^{\frac{3}{2}} y^{*}, t \in(0, T) \\
& q^{T}(T)=-R y^{*}(T) .
\end{aligned}
$$

(For existence in (2.13) we use the fact that $q^{T}(T) \in W^{\prime} \subset V^{\prime}$ and apply the standard existence theory for linear evolution equations.)

By (2.12) and the unique continuous property for the Stokes equation

$$
q_{t}-\left(\nu A+A_{0}\right)^{*} q=0 \text { in } Q ; q=0 \text { on } \Sigma
$$

(which is a consequence of the Carleman inequality established in [14,15] for the Stokes equation) it follows that $q^{T}=q^{T^{\prime}}$ on $(0, T)$ for $0<T<T^{\prime}$. Hence $q^{T}=q$ is independent of $T$ and so $(2.12,2.13)$ extend to all of $R^{+}$. Moreover, we have

$$
R y^{0}=-q^{T}(0)
$$

Indeed for all $y^{0}, z^{0} \in D\left(A^{\frac{1}{4}}\right)$ we have by $(2.5)^{\prime}$ that

$$
\begin{aligned}
\varphi\left(y^{0}\right)-\varphi\left(z^{0}\right) \leq & \int_{0}^{T}\left(A^{\frac{3}{4}} y^{*}(t), A^{\frac{3}{4}}\left(y^{*}(t)-z^{*}(t)\right)+\left(u^{*}(t), u^{*}(t)-v^{*}(t)\right)\right) \mathrm{d} t \\
& +\left(R y^{*}(T), y^{*}(T)-z^{*}(T)\right)
\end{aligned}
$$

where $\left(z^{*}, v^{*}\right)$ is the optimal pair corresponding to $z^{0}$. On the other hand, by $(2.12)$ and $(2.13)$ we see that

$$
\frac{\mathrm{d}}{\mathrm{d} t}\left(q^{T}(t), y^{*}(t)-z^{*}(t)\right)=\left(A^{\frac{3}{4}} y^{*}(t), A^{\frac{3}{4}}\left(y^{*}(t)-z^{*}(t)\right)+\left(u^{*}(t), u^{*}(t)-v^{*}(t)\right)\right) .
$$

Integrating on $(0, T)$ and substituting into the above inequality we obtain that

$$
\varphi\left(y^{0}\right)-\varphi\left(z^{0}\right) \leq-\left(q^{T}(0), y^{0}-z^{0}\right)
$$

which implies (2.14) as desired. 
By (2.12) we infer that

$$
q(t)=-R y^{*}(t), \forall t \geq 0
$$

and this implies (2.8) as claimed.

By (2.4) we have also that

$$
\begin{aligned}
\frac{\mathrm{d}}{\mathrm{d} t}\left\|y^{*}(t)\right\|^{2}+2 \nu\left|A y^{*}(t)\right|^{2} & +2 b\left(y_{e}, y^{*}(t), A y^{*}(t)\right) \\
& +2 b\left(y^{*}(t), y_{e}, A y^{*}(t)\right) \leq 2\left|m q(t) \| A y^{*}(t)\right|, \text { a.e. } t \in(0, T) .
\end{aligned}
$$

Since as noticed earlier

$$
\left|b\left(y_{e}, y, A y\right)\right|+\left|b\left(y, y_{e}, A y\right)\right| \leq C\left|y_{e}\right|_{2}\|y\||A y|
$$

we find the estimate

$$
\left\|y^{*}(t)\right\|^{2}+\int_{0}^{t}\left|A y^{*}(t)\right|^{2} \mathrm{~d} t \leq C_{T}\left\|y^{0}\right\|^{2}, \forall t \in(0, T) .
$$

On the other hand, coming back to equation (2.13) and substituting $z=A^{-\frac{1}{2}} q$ we obtain

$$
\frac{\mathrm{d}}{\mathrm{d} t} z-\nu A z-A^{-\frac{1}{2}} A_{0}^{*} A^{\frac{1}{2}} z=A y^{*} .
$$

Noticing that

$$
\left|\left(A^{-\frac{1}{2}} A_{0}^{*} A^{\frac{1}{2}} z, A z\right)\right| \leq\left|b\left(y_{e}, A^{\frac{1}{2}} z, A^{\frac{1}{2}} z\right)\right|+\left|b\left(A^{\frac{1}{2}} z, y_{e}, A^{\frac{1}{2}} z\right)\right| \leq C|A z|\|z\|\left|y_{e}\right|_{2}
$$

and recalling that $A y^{*} \in L^{2}(0, T ; H)$ we see by $(2.17)$ that $z \in C_{w}([0, T) ; V)$. Hence $q \in C([0, T) ; H)$ and so $-q(0)=R y^{0} \in H$. Then (2.9) follows by the closed graph theorem.

Next by $(2.5)^{\prime}$ we have

$$
\varphi\left(y^{*}(t)\right)=\frac{1}{2} \int_{t}^{\infty}\left(\left|A^{\frac{3}{4}} y^{*}\right|^{2}+\left|u^{*}\right|^{2}\right) \mathrm{d} s, \forall t \geq 0
$$

and therefore

$$
\left(R y^{*}(t), \frac{\mathrm{d} y^{*}}{\mathrm{~d} t}(t)\right)+\frac{1}{2}\left|A^{\frac{3}{4}} y^{*}(t)\right|^{2}+\frac{1}{2}\left|m R y^{*}(t)\right|^{2}=0 \text {, a.e. } t>0 \text {. }
$$

Since $|P(m R y)| \leq C\|y\|, \forall y \in V$ we see that the operator $\nu A+A_{0}+P(m R)$ with the domain $D(A)$ generates a $C_{0}-$ semigroup on $H$ (this is just the flow $y_{0} \rightarrow y^{*}(t)$ ). This implies that

$$
A y^{*}, A_{0} y^{*}, P\left(m R y^{*}\right) \in C([0, \infty) ; H)
$$

and in virtue of (2.18) and (2.8) this yields

$$
-\left(R y^{*}(t), \nu A y^{*}(t)+A_{0} y^{*}(t)\right)-\frac{1}{2}\left|m R y^{*}(t)\right|^{2}+\frac{1}{2}\left|A^{\frac{3}{4}} y^{*}(t)\right|^{2}=0, \forall t \geq 0
$$

thereby completing the proof.

Remark 1. It is easily seen that the equation (2.11) has a unique self-adjoint solution $R$ satisfying conditions (2.9) and (2.10). This is an immediate consequence of the fact that any such a solution stabilizes system (2.4) via feedback law (2.8). The general problem of uniqueness in equation (2.11) with bounded right hand side is studied in $[8]$ but the arguments extend in our case too. 


\section{Stabilization of the Navier-Stokes equation}

Theorem 1 below is the main result of this paper.

Theorem 1. Let $d=2,3$ and let $R$ be the operator defined in Proposition 1 . Let $\left(y_{e}, p_{e}\right)\left(\left(H^{3}(\Omega)\right)^{d} \cap V\right) \times H^{1}(\Omega)$ be a steady-state solution to equation (1.1). Then the feedback controller

$$
u=-m R\left(y-y_{e}\right)
$$

exponentially stabilizes $y_{e}$ in a neighbourhood $\mathcal{V}=\left\{y_{0} \in W ;\left\|y_{0}-y_{e}\right\|_{W}<\rho\right\}$ of $y_{e}$. More precisely, for each $y_{0} \in \mathcal{V}$ there is a weak solution $y \in L_{\mathrm{loc}}^{\infty}\left(R^{+} ; H\right) \cap L_{\mathrm{loc}}^{2}\left(R^{+} ; V\right)$ to closed loop system

$$
\begin{aligned}
& \frac{\mathrm{d} y}{\mathrm{~d} t}+\nu A y+B y+P\left(m R\left(y-y_{e}\right)\right)=P f_{0}, t>0 \\
& y(0)=y_{0}
\end{aligned}
$$

such that

$$
\begin{aligned}
& \int_{0}^{\infty}\left|A^{\frac{3}{4}}\left(y(t)-y_{e}\right)\right|^{2} \mathrm{~d} t \leq C\left\|y_{0}-y_{e}\right\|_{W}^{2} \\
& \left|y(t)-y_{e}\right| \leq C \mathrm{e}^{-\gamma t}\left\|y_{0}-y_{e}\right\|_{W}, \forall y_{0} \in \mathcal{V}
\end{aligned}
$$

for some $\gamma>0$.

Proof. As seen earlier we may reduce the problem to that of stability of the null solution to corresponding closed loop system (2.2), i.e.,

$$
\begin{aligned}
& \frac{\mathrm{d} y}{\mathrm{~d} t}+\nu A y+A_{0} y+B y+P(m R y)=0, t>0 \\
& y(0)=y^{0} .
\end{aligned}
$$

We consider the approximating equation

$$
\begin{aligned}
& \frac{\mathrm{d} y_{N}}{\mathrm{~d} t}+\nu A y_{N}+A_{0} y_{N}+B_{N} y_{N}+P\left(m R y_{N}\right)=0, \\
& y_{N}(0)=y^{0}
\end{aligned}
$$

where

We note that

$$
B_{N} y=B y \text { if }\|y\| \leq N, B_{N} y=\frac{N^{2}}{\|y\|^{2}} B y \text { if }\|y\|>N
$$

and by (2.9) we have

$$
\left(B_{N} y-B_{N} z, y-z\right) \geq-\varepsilon\|y-z\|^{2}-C_{N, \varepsilon}|y-z|^{2}, \forall y, z \in V
$$

$$
|P(m R y)| \leq C\|y\|, \forall y \in V .
$$

Then arguing as in $[4,6]$ it follows that the operator $\mathcal{A}_{N}=\nu A+A_{0}+B_{N}+P(m R)$ with the domain $D(A)$ is $m$ quasi-accretive in $H$ (i.e., $\lambda I+\mathcal{A}_{N}$ is $m$-accretive in $H$ for some $\left.\lambda>0\right)$. Thus for each $y^{0} \in D(A)$ equation (3.5) has a unique solution $y_{N} \in W_{\mathrm{loc}}^{1, \infty}\left(R^{+} ; H\right) \cap L_{\mathrm{loc}}^{\infty}\left(R^{+} ; D(A)\right)$. (If $y^{0} \in V$ then $y_{N} \in W_{\mathrm{loc}}^{1,2}\left(R^{+} ; H\right)$ $\cap L_{\text {loc }}^{2}\left(R^{N} ; D(A)\right) \cap C\left(R^{+} ; V\right)$.) Also the following estimate holds

$$
\left|y_{N}(t)\right|^{2}+\int_{0}^{t}\left(\left\|y_{N}^{\prime}\right\|_{V^{\prime}}^{\frac{4}{3}}+\left\|y_{N}\right\|^{2}\right) \mathrm{d} s \leq C_{T}, \forall t \in(0, T),
$$

where $y_{N}^{\prime}=\frac{\mathrm{d} y_{N}}{\mathrm{~d} t}$ and $C_{T}$ is independent of $N$. 
This implies that there is a subsequence $N \rightarrow \infty$ such that on each finite interval $(0, T)$,

$$
y_{N} \longrightarrow y \text { weak star in } L^{\infty}(0, T ; H) \text {, weakly in } L^{2}(0, T ; V)
$$

strongly in $L^{2}(0, T ; H)$

where $y \in L_{\text {loc }}^{2}\left(R^{+} ; V\right) \cap C_{w}\left(R^{+} ; H\right), \frac{\mathrm{d} y}{\mathrm{~d} t} \in L_{\mathrm{loc}}^{\frac{3}{4}}\left(R^{+} ; V^{\prime}\right)$ is a weak solution to equation (3.4). (See e.g. [10,17] for the definition of the weak solution.)

Now we multiply equation (3.5) by $R y_{N}$ and use equation (2.11) to obtain after some calculation that

$$
\frac{\mathrm{d}}{\mathrm{d} t}\left(R y_{N}(t), y_{N}(t)\right)+\left|m R y_{N}(t)\right|^{2}+\left|A^{\frac{3}{4}} y_{N}(t)\right|^{2}=-2\left(B_{N} y_{N}(t), R y_{N}(t)\right), \text { a.e. } t>0 \text {. }
$$

On the other hand, recalling that (see e.g. $[10,17]$ )

$$
|b(y, z, w)| \leq C|y|_{m_{1}}|z|_{m_{2}+1}|w|_{m_{3}}, m_{1}+m_{2}+m_{3} \geq \frac{d}{2}
$$

it follows by Proposition 1 that

$$
\begin{aligned}
\left|\left(B_{N} y_{N}, R y_{N}\right)\right| & \leq \inf \left(1, \frac{N^{2}}{\left\|y_{N}\right\|^{2}}\right)\left|b\left(y_{N}, y_{N}, R y_{N}\right)\right| \\
& \leq C\left|y_{N}\right|_{1}\left|y_{N}\right|_{\frac{3}{2}}\left|R y_{N}\right| \leq C\left\|y_{N}\right\|\left|A^{\frac{3}{4}} y_{N}\right|\left|R y_{N}\right| \\
& \leq\left|A^{\frac{3}{4}} y_{N}\right|\left\|y_{N}\right\|^{2} \leq C\left|A^{\frac{3}{4}} y_{N}\right|^{2}\left(R y_{N}, y_{N}\right)^{\frac{1}{2}}
\end{aligned}
$$

because by interpolation we have

$$
\|y\|^{2} \leq\left|A^{\frac{3}{4}} y\right|\left|A^{\frac{1}{4}} y\right| \leq C\left|A^{\frac{3}{4}} y\right|(R y, y)^{\frac{1}{2}} .
$$

We set

$$
E=\left\{y^{0} \in W ;\left(R y^{0}, y^{0}\right)<\rho\right\} .
$$

Then by (3.8) and (3.9) we see that for $\rho$ sufficiently small and independent of $N$ and $y^{0} \in E$ we have

$$
\frac{\mathrm{d}}{\mathrm{d} t}\left(R y_{N}(t), y_{N}(t)\right)+\frac{1}{2}\left|A^{\frac{3}{4}} y_{N}(t)\right|^{2} \leq 0, \text { a.e. } t>0 \text {. }
$$

By (2.10) this yields

$$
\frac{\mathrm{d}}{\mathrm{d} t}\left(R y_{N}(t), y_{N}(t)\right)+\gamma\left(R y_{N}(t), y_{N}(t)\right) \leq 0 \text {, a.e. } t>0
$$

for some positive constant $\gamma$ independent of $N$ and

$$
\int_{0}^{\infty}\left|A^{\frac{3}{4}} y_{N}(t)\right|^{2} \mathrm{~d} t \leq 2\left(R y^{0}, y^{0}\right) .
$$

Then again using (2.10) this yields

$$
\left|y_{N}(t)\right| \leq\left\|y_{N}(t)\right\|_{W} \leq C\left\|y^{0}\right\|_{W} \mathrm{e}^{-\gamma t}, \forall t \geq 0
$$


for some $\gamma>0$ and $C>0$ independent of $N$. Then recalling that in virtue of (3.7) we may assume that $\left\{y_{N}\right\}$ is strongly convergent in $H$ a.e. on $R^{+}$, letting $N$ tend to $+\infty$ it follows by (3.7) that

$$
|y(t)| \leq C\left\|y^{0}\right\|_{W} \mathrm{e}^{-\gamma t}, \forall t \geq 0
$$

and so (3.3) follows for $\mathcal{V}=E+y_{e}$. This completes the proof.

We note that if $d=2$ then the solution $y$ to closed loop system (3.2) is a strong solution and unique for each $y_{0} \in \mathcal{V}$.

Remark 2. The same linearization technique can be used to solve the local $H^{\infty}$ problem for the Navier-Stokes equation with exogeneous disturbances. This problem was solved in [5] via differential game approach.

Remark 3. It is readily seen that under conditions of Theorem 1 the feedback controller $u=-\psi\left(m R\left(y-y_{e}\right)\right)$ is asimptotically stable for all continuous mappings $\psi: H \rightarrow H$ such that

$$
(\psi(z), z) \geq \frac{1+\delta}{2}|z|^{2}, \forall z \in H
$$

for some $\delta>0$. This follows as above by multiplying the closed loop equation (2.4) with the feedback control $u=$ $-\psi(m R y)$ by $R y$ and using (2.11) and (3.10).

This can be seen as a robustness property of the feedback controller (3.1) with respect to static perturbations in the input.

\section{REFERENCES}

[1] F. Abergel and R. Temam, On some control problems in fluid mechanics. Theoret. Comput. Fluid Dynam. 1 (1990) 303-325.

[2] V. Barbu, Mathematical Methods in Optimization of Differential Systems. Kluwer, Dordrecht (1995).

[3] V. Barbu, Local controllability of Navier-Stokes equations. Adv. Differential Equations 6 (2001) 1443-1462.

[4] V. Barbu, The time optimal control of Navier-Stokes equations. Systems E Control Lett. 30 (1997) 93-100.

[5] V. Barbu and S. Sritharan, $H^{\infty}$-control theory of fluid dynamics. Proc. Roy. Soc. London 454 (1998) 3009-3033.

[6] V. Barbu and S. Sritharan, Flow invariance preserving feedback controller for Navier-Stokes equations. J. Math. Anal. Appl. 255 (2001) 281-307.

[7] Th.R. Bewley and S. Liu, Optimal and robust control and estimation of linear path to transition. J. Fluid Mech. 365 (1998) 305-349.

[8] A. Bensoussan, G. Da Prato, M.C. Delfour and S.K. Mitter, Representation and Control of Infinite Dimensional Systems. Birkhäuser, Boston, Bassel, Berlin (1992).

[9] C. Cao, I.G. Kevrekidis and E.S. Titi, Numerical criterion for the stabilization of steady states of the Navier-Stokes equations. Indiana Univ. Math. J. 50 (2001) 37-96.

[10] P. Constantin and C. Foias, Navier-Stokes Equations. University of Chicago Press, Chicago, London (1989).

[11] J.M. Coron, On the controllability for the 2-D incompresssible Navier-Stokes equations with the Navier slip boundary conditions. ESAIM: COCV 1 (1996) 33-75.

[12] J.M. Coron, On the null asymptotic stabilization of the 2-D incompressible Euler equations in a simple connected domain. SIAM J. Control Optim. 37 (1999) 1874-1896.

[13] J.M. Coron and A. Fursikov, Global exact controllability of the 2-D Navier-Stokes equations on a manifold without boundary. Russian J. Math. Phys. 4 (1996) 429-448.

[14] O.A. Imanuvilov, Local controllability of Navier-Stokes equations. ESAIM: COCV 3 (1998) 97-131.

[15] O.A. Imanuvilov, On local controllability of Navier-Stokes equations. ESAIM: COCV 6 (2001) 49-97.

[16] I. Lasiecka and R. Triggianni, Control Theory for Partial Differential Equations: Continuous and Approximation Theories, Encyclopedia of Mathematics and its Applications. Cambridge University Press (2000).

[17] R. Temam, Navier-Stokes Equations and Nonlinear Functional Analysis. SIAM Philadelphia (1983). 